\title{
Determination of Optical Constants and Thickness of Nanostructured ZnO Film by Spin Coating Technique
}

\author{
R. R. Ghimire, Y. P. Dahal, K. B. Rai and S. P. Gupta
}

Journal of Nepal Physical Society

Volume 7, Issue 2, June 2021

ISSN: 2392-473X (Print), 2738-9537 (Online)

Editors:

Dr. Binod Adhikari

Dr. Bhawani Joshi

Dr. Manoj Kumar Yadav

Dr. Krishna Rai

Dr. Rajendra Prasad Adhikari

Mr. Kiran Pudasainee

JNPS, 7 (2), 119-125 (2021)

DOI: https://doi.org/10.3126/jnphyssoc.v7i2.38632

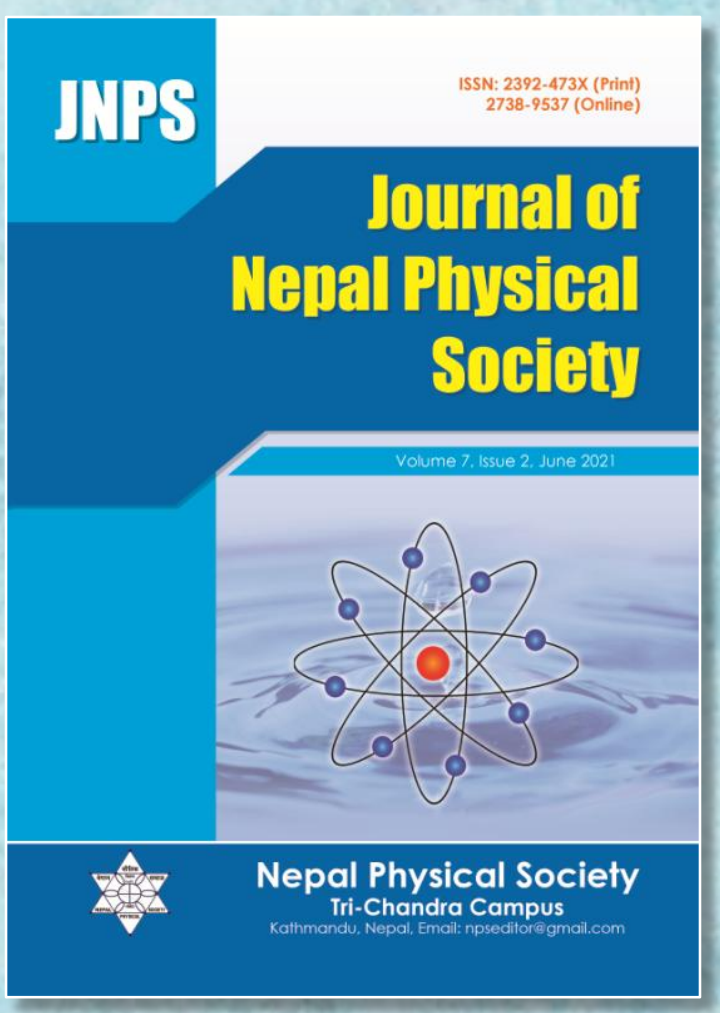

Published by:

Nepal Physical Society

P.O. Box: 2934

Tri-Chandra Campus

Kathmandu, Nepal

Email: nps.editor@gmail.com 


\title{
Determination of Optical Constants and Thickness of Nanostructured ZnO Film by Spin Coating Technique
}

\author{
R. R. Ghimire ${ }^{1}$, Y. P. Dahal ${ }^{2}$, K. B. Rai ${ }^{1}$ and S. P. Gupta ${ }^{1, *}$ \\ ${ }^{1}$ Department of Physics, Patan Multiple Campus, Tribhuvan University, Lalitpur \\ ${ }^{2}$ Department of Physics Bhaktapur Multiple Campus, Tribhuvan University, Bhaktapur \\ *Corresponding Email: suresh.gupta @ pmc.edu.np
}

Received: 05 May, 2021; Revised: 13 June, 2021; Accepted: 25 June, 2021

\begin{abstract}
In this report, we have investigated optical constants and thickness of nanostructured $\mathrm{ZnO}$ films grown on a glass substrate by sol-gel spin coating technique using zinc acetate as precursor. Optical constants such as complex refractive index $\tilde{n}$ and dielectric constant $\epsilon$ determined from the transmittance spectrum in the ultraviolet, visible, near infrared (UV-VIS, NIR) region by envelope method. The value of refractive index decreases from 2.34 to 1.86 and extinction coefficient increases from 0.28 to 0.64 with increasing wavelength. The decreasing behavior of refractive index is attributed due to the increase in transmission and decrease in absorption coefficient with increasing wavelength. The film exhibits reasonably high transmittance $(>80 \%)$ in the visible region. Absorbance coefficient $\alpha$ and film thickness (d) were calculated from the interference of fringes of transmittance spectrum. The band gap and thickness of the film were found $3.02 \mathrm{eV}$ and $275 \mathrm{~nm}$, respectively. The thickness of the film measured by envelope method is validated with cross-section micrograph of SEM images which is about $285 \mathrm{~nm}$. The real part of the dielectric function of nanostructured $\mathrm{ZnO}$ decreases with increasing wavelength where as the imaginary part of dielectric constant increases with increasing wavelength. The observed high value of refractive index $\mathrm{n}$ and real part of dielectric constant $\epsilon$ at lower wavelength is due to band edge absorption of carriers. The dispersion relation shows the increase of complex refractive index and dielectric constant at the high frequency regime is due to the discharging of defect levels using optical excitation of carriers in the visible region.
\end{abstract}

Keywords: Transparent conducting oxide, Grain boundary, Dielectric constants, Scanning electron microscope, UV-visible Spectroscopy.

\section{INTRODUCTION}

Recently, transparent conducting oxides (TCO) have been widely studied because of its excellent optoelectronic applications. Among various TCOs, $\mathrm{ZnO}$ is one of the most promising materials for the fabrication of next generation optoelectronic devices in the UV region and optical and display devices. $\mathrm{ZnO}$ is a wide band gap $\left(\mathrm{E}_{\mathrm{g}}=3.3 \mathrm{eV}\right)$ semiconductor with high exciton binding energy $(60 \mathrm{meV})$. The devices based on $\mathrm{ZnO}$ nanostructure can operate at high temperature, high electric and magnetic field and also scale down to the nanoscale regime with fascinating optoelectronic properties. The high value of optical transmittance in visible region and electrical conductance at ambient temperature make $\mathrm{ZnO}$ an important material for possible application in blue and ultraviolet (UV) light emitters [1,2], photovoltaic devices [1,2], surface acoustic wave devices [1,2,3], gas sensors $[1,2,4,5]$, light emitting diode (LED) [4,5,6], Varistors [1], heat mirrors, conducting coating in aircraft glass and transparent electrodes $[3,4,5]$.

The various most intensively studied techniques to grow the semiconductor thin film are spin coating, spray pyrolysis, dip coating, and an electrochemical method using chemical route $[6,7,8,9,10]$. Beside this some physical methods such as thermal evaporation, pulsed laser deposition and sputtering methods are 
used to grow high quality $\mathrm{ZnO}$ films on different substrate even at low temperature flexible polyamide or textile substrates [11]. Sol-gel spin coating technique is an excellent method for the deposition of nanostructured $\mathrm{ZnO}$ thin film using the acetate precursor solution with a sol stabilizing agent like amine $[1,4,5]$. The spin speed and number of coatings primarily controlled the film thickness and grain size, which shows the possibility to tune the size dependent material properties.

The accurate knowledge of crystal structure, film thickness and some optical constants such as band gap, complex refractive index and dielectric constant is critically important for the fabrication of high quality optoelectronic devices. The optical characterization of the films gives the physical parameters such as band gap, refractive index, dielectric constants and film thickness. The current methods for determining the optical constants of transparent thin films usually based on highly advanced expensive computerized technique. In our previous study $[4,9]$ we have investigated the role of aluminium doping and controlled of defect states lying in visible region which enhanced the carrier mobility as well as the photo response of nanostructured $\mathrm{ZnO}$ film. On the other hand grain and grain boundaries have the major role in carrier generation, recombination and transport properties of nanostructured films.

In this report, we employ a non destructive technique to determine optical constants and film thickness, which is highly recommended in scientific research and industrial production of granular TCO. Here we have calculated film thickness, absorption coefficient and optical dispersion relation of pristine $\mathrm{ZnO}$ film grown on glass substrate using the envelope method. Transmittance spectra measured using spectrophotometer used to evaluate absorption coefficient, optical band gap, complex refractive index, dielectric constants and film thickness. There are few papers which report the determination of optical constants and film thickness using non destructive Swanepoel envelope method $[8,12,14,15]$, but there is no report which compares the film thickness obtained from scanning electron micrograph with the thickness calculated from transmittance spectra using envelope method. Therefore, in this paper, we report a thickness measurement using transmission spectra and validated with a cross-section image of $\mathrm{ZnO}$ films. We have also studied the wavelength dependent complex refractive index and dielectric function of undoped nanostructured $\mathrm{ZnO}$ films in $\mathrm{UV}$ and visible illumination region. The high transmission and low absorption in visible and infrared (IR) region urges to decrease the refractive index $n$ and dielectric constant $\epsilon$ within this wavelength region. The critical role to modulate the optical constants under illumination is essentially by carrier generation and discharging the grain boundary defect levels by optically excited carriers [9].

\section{EXPERIMENT}

Undoped $\mathrm{ZnO}$ thin films were prepared by sol-gel spin coating method. Zinc acetate dihydrate, ethanol and diethanolamine (DEA) were used as starting material, solvent and stabilizer, respectively. Zinc acetate dihydrate was first dissolved in a mixture of ethanol and DEA solution at room temperature. The molar ratio of DEA to zinc acetate was maintained at approximately 1.0 and the molar concentration of zinc acetate was fixed at $0.1 \mathrm{M}$. The solution was stirred at $60^{\circ} \mathrm{C}$ for $2 \mathrm{hrs}$ to yield a clear and homogeneous solution, which served as the precursor solution. The precursor solution was filtered through filter paper and dropped onto glass substrates, which were rotated at $3400 \mathrm{rpm}$ for $30 \mathrm{~s}$. The coated film is first dried at $120^{\circ} \mathrm{C}$ for 5 minutes and then heated to $450^{\circ} \mathrm{C}$ for 10 minutes. The process of coating and drying is repeated several times to get the required thickness. The experimental procedure is described in the flow chart shown in Fig. 1.

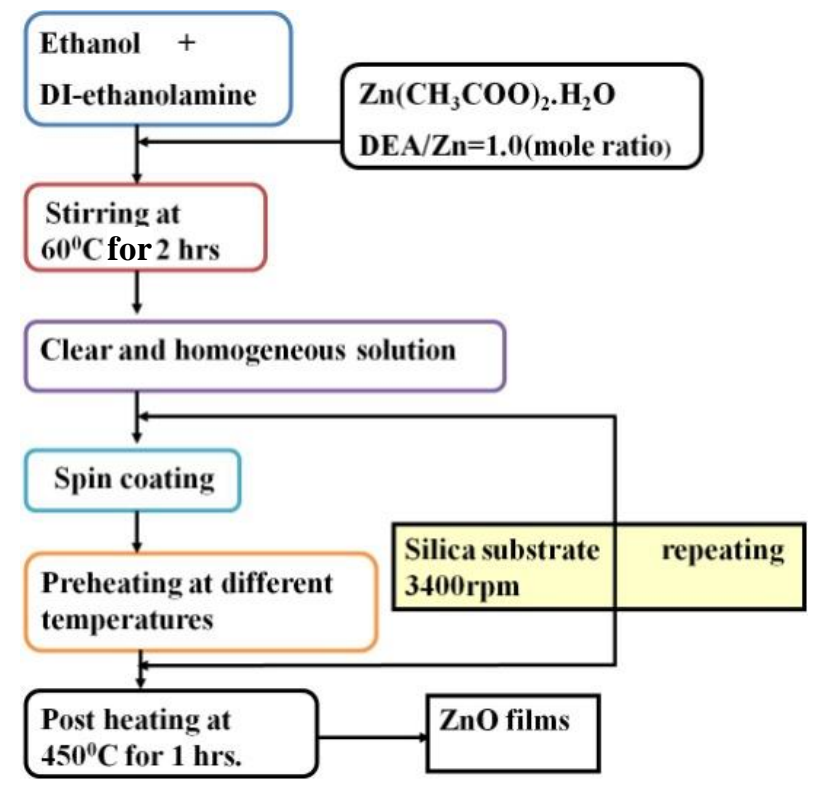

Fig. 1: Flow chart to deposit a ZnO film by spin coating. 
The optical properties of $\mathrm{ZnO}$ films were carried out with Ultraviolet-visible Spectrometer (Ocean Insight model no: FLAME-T-UV-VIS). The optical transmittance at normal incidence was recorded in the wavelength range of $375 \mathrm{~nm}-1300$. Swanepoel's envelope method $[10,14,16,17]$ was employed to evaluate the optical constants such as the refractive index $\mathrm{n}$, extinction coefficient $k$ and absorption coefficient $\alpha$ from transmittance spectra. The thickness of the $\mathrm{ZnO}$ films was determined from interference fringes of transmittance data and validated with the cross-section SEM micrograph.

\section{RESULTS AND DISCUSSION}

\section{Determination of optical band gap and thickness of $\mathrm{ZnO}$ film}

Fig. 2 (a) depicts the variation of optical transmittance (T\%) of $\mathrm{ZnO}$ thin films in the wavelength region $375 \mathrm{~nm}$ to $1300 \mathrm{~nm}$. The average transmittance of the film, including substrate in the visible range is observed over $84 \%$. This high transmittance value suggests that the film is better crystalline and can be used as a TCO and solar cell electrodes. An excellent surface quality and homogeneity of the film is also confirmed by the appearance of interference fringes in the transmittance spectra which appears due to the reflection from the film surface without much absorption and scattering in the bulk of the film [16]. The value of transmittance is suddenly dropped close to zero when the wavelength falls below $400 \mathrm{~nm}$ because of band gap absorption of $\mathrm{ZnO}$. The optical constants such as refractive index $\mathrm{n}$, absorption coefficient $\alpha$, extinction coefficient $\mathrm{k}$ and band gap $\left(E_{g}\right)$ and film thickness were determined from the transmittance spectrum by envelope method [17]. (a)

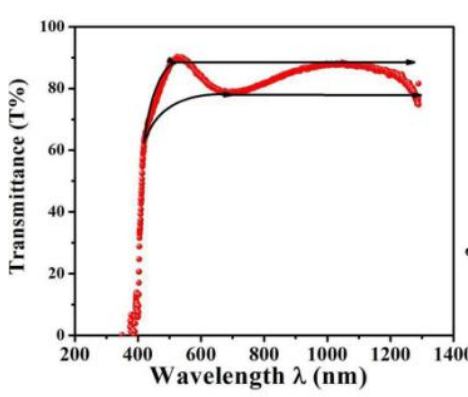

(b)

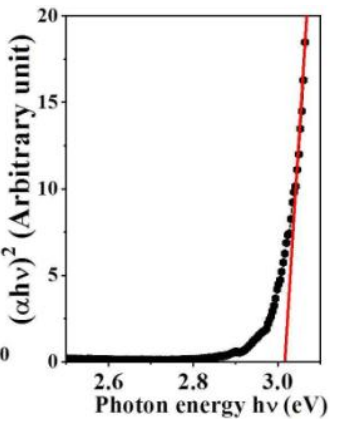

Fig. 2: (a) Transmittance spectrum of undoped $\mathrm{ZnO}$ films annealed at $450^{\circ} \mathrm{C}$ (b) Plot of $(\alpha \mathrm{hv})^{2}$ as a function of photon energy
These optical constants are evaluated outside the region of fundamental band gap $\left(h v=E_{g}\right)$. For this we have made envelope in the interference region (weak and medium absorption region) in the transmittance curve as shown in Fig. 2 (a).

The absorption coefficient $\alpha$ and the extinction coefficient $\kappa$ can be calculated as, $[13,16]$

$$
\begin{gathered}
\alpha=\frac{1}{d} \ln \frac{(n-1)\left(n-n_{S}\right)\left[\left(T_{\max } / T_{\text {min }}\right)^{1 / 2}+1\right]}{(n+1)\left(n+n_{S}\right)\left[\left(T_{\max } / T_{\min }\right)^{1 / 2}-1\right]} \ldots \text { (1) } \\
\kappa=\frac{\alpha \lambda}{4 \pi} \ldots(2)
\end{gathered}
$$

Where $\alpha$ is the absorbance coefficient and $d$ is the film thickness and $\lambda_{1}$ and $\lambda_{2}$ are the wavelength at two adjacent maxima or minima.

An optical band gap of nanostructured $\mathrm{ZnO}$ film is calculated from the fundamental absorption edge of the films which corresponds to the transition of electrons from the valence band to the conduction band.

Material like $\mathrm{ZnO}$ which has direct transition, the absorption gap $\left(\mathrm{E}_{\mathrm{g}}\right)$ can be expressed by $[2,3,4,12]$

$$
\alpha h v=\left[A\left(h v-E_{g}\right)\right]^{1 / 2}
$$

Where $\mathrm{A}$ is a constant, $h v$ is the photon energy and $\mathrm{Eg}$ is the optical energy band of $\mathrm{ZnO}$. Fig. 2 (b) depicts the plot of $(\alpha h v)^{2}$ versus photon energy $(\mathrm{h} v)$ for $\mathrm{ZnO}$ sample. The values for $(\alpha h v)^{2}$ and $(h v)$ were determined from transmittance data for different wavelength. The optical band gap $\left(E_{g}\right)$ of the films was determined by extrapolating the linear portion of $(\mathrm{ahv})^{2}$ versus $h v$ curve to $(a h v)^{2}=0$. The calculated value of the band gap in $\mathrm{ZnO}$ film was found to be $3.02 \mathrm{eV}$, which is shown in table 1 . The obtained value of band gap is slightly less than the most of reported value which may arise due to the large grain size and impurities' effect [4].

The thickness of the film was calculated using following relation $[13,14]$

$$
d=\frac{\lambda_{1} \lambda_{2}}{\left(n_{1}^{1} \lambda_{1}-n_{1}^{11} \lambda_{2}\right)}
$$

Where, $n_{1}^{1}$ and $n_{2}^{11}$ are the refractive indices at two adjacent maxima (or minima) corresponding to 
the wavelengths $\lambda_{1}$ and $\lambda_{2}$. The thickness of the zinc oxide film calculated by this method was found to be $275 \mathrm{~nm}$. This is very close to the thickness measured from SEM image $(290 \mathrm{~nm})$.

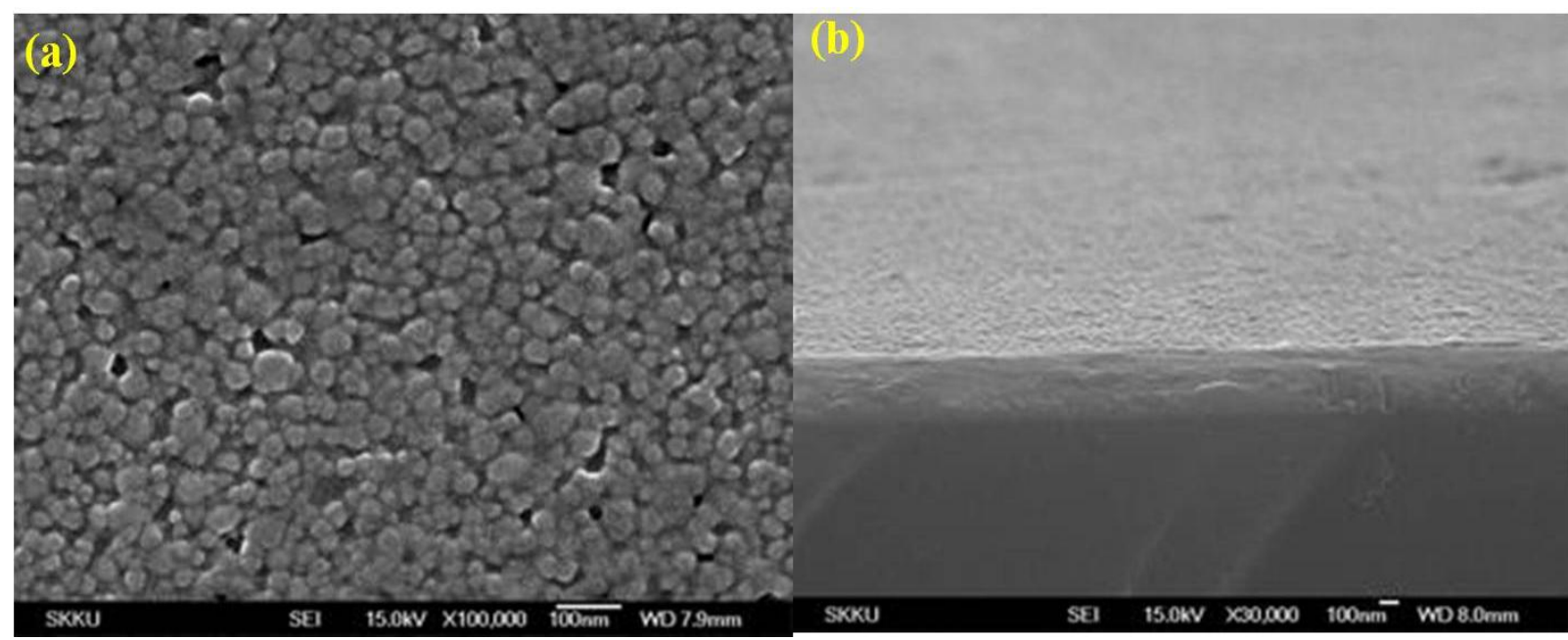

Fig. 3: SEM Micrograph of undoped ZnO films annealed at $450^{\circ} \mathrm{C}$ (a) Surface view of (b) cross-section view

Fig. 3 (a) shows the surface view of the Scanning Electron micrograph (SEM) of the $\mathrm{ZnO}$ films. The film consists with large no. of grains and grain boundaries. The porosity and voids of the films are clearly observed in this image. The average grain size of the film is observed $\sim 30$ nm. Fig. 3 (b) shows the SEM image of a crosssection of undoped $\mathrm{ZnO}$ film and the thickness of the film is $\sim 285 \mathrm{~nm}$. This value of thickness is very close to the thickness measured by envelope method.

\section{Determination of complex refractive index and} Cauchy's parameters of $\mathrm{ZnO}$ films

The variation of refractive index $n$ and extinction coefficient $\kappa$ with wavelength in the region $400 \mathrm{~nm}$ $-1100 \mathrm{~nm}$ is shown in Fig. 4.

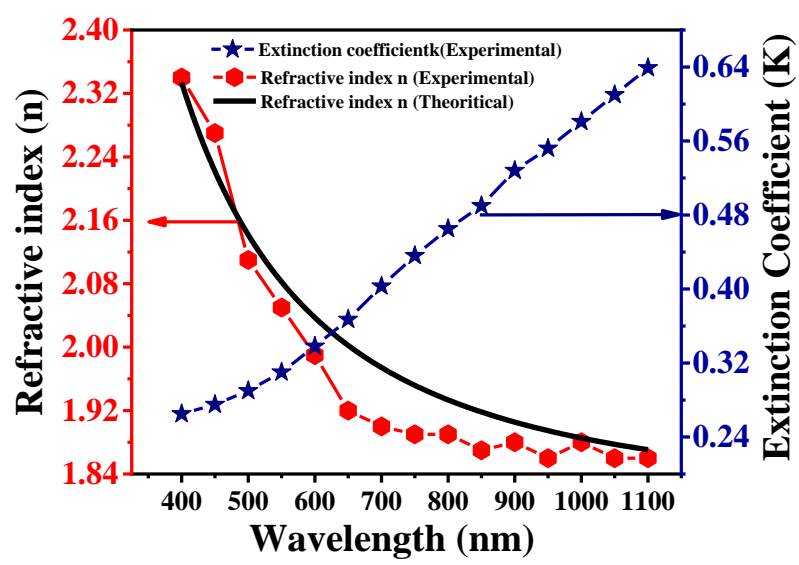

Fig. 4: $n$ and $k$ versus $\lambda$ of undoped $\mathrm{ZnO}$ film

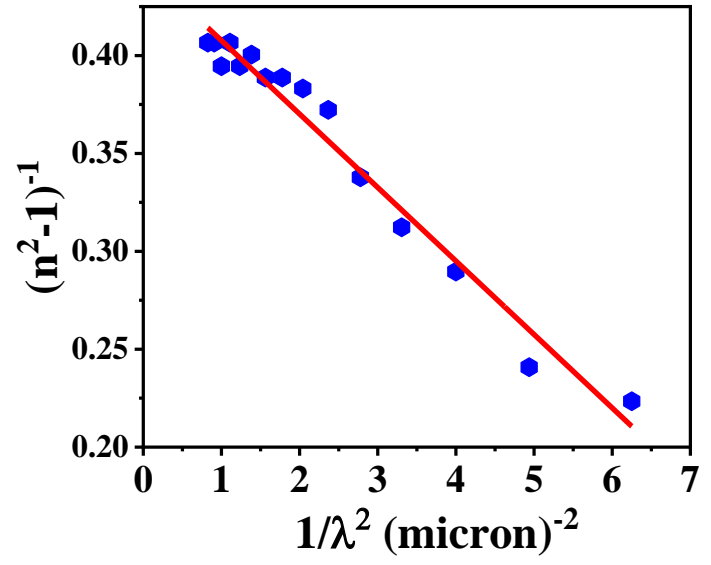

Fig. 5: Plot of $\left(n^{2}-1\right)^{-1}$ versus $\lambda^{-2}$

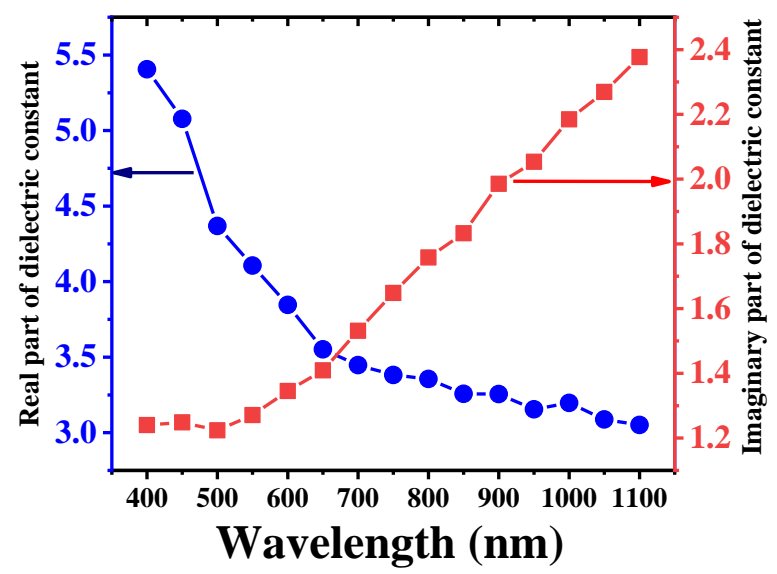

Fig. 6: $\lambda$ versus $n$ of nanostructured $\mathrm{ZnO}$ film 
The dispersion of refractive index $(n)$ of the film along the Cauchy's constants was theoretically observed in $\mathrm{ZnO}$ by the following relation $[10,12]$

$$
n=A+\frac{B}{\lambda^{2}}
$$

Where, $A$ and $B$ are Cauchy's parameter and $\lambda$ is the wavelength of light interacted with the film. The experimental graph is very closely matched with simulated curve. Fig. 4 shows the refractive index $n$ vs. wavelength $\lambda$ (dispersion relation of refractive index : solid black curve shows the theoretical and red dashed curve shows with experimental plot). Experimentally the value of refractive index is calculated using the fringe envelop method:

$$
\begin{gathered}
n=\sqrt{N+\sqrt{N^{2}+n_{S}^{2}}} \ldots(6) \\
\text { Where } N=2 n_{S}\left(\frac{T_{M}+T_{m}}{T_{M} T_{m}}\right)+\frac{1+n_{s}^{2}}{2} \ldots
\end{gathered}
$$

$T_{M}$ and $T_{m}$ are maximum and minimum transmission respectively, on the envelope at a certain $\lambda$, and refractive index $n_{\mathrm{s}}$ of the substrate.

The envelopes connecting the interference maxima and minima are considered to be continuous functions of the wavelength $\lambda$. Therefore, for each maximum of the transmission curves $\left(\mathrm{T}_{\mathrm{M}}\right)$ corresponding minimum $\left(T_{m}\right)$ may be determined at the same $\lambda$, and vice versa. Fig. 4 shows the plot of complex refractive index $\tilde{n}=n+i k$ of the film with wavelength $\lambda$, where $n(w)$ is the real part and $k(w)$ is the imaginary part (extinction coefficient) of refractive index $\tilde{n}$.

The real part of complex refractive index varies from a minimum of 1.86 to a maximum of 2.34 . The value of the real part of the refractive index decreases with increasing the wavelength and the imaginary part of complex refractive index i.e. Extinction coefficient is increasing with increasing wavelength. The value of $n$ is first steeply down up to $600 \mathrm{~nm}$ and then gradually decreasing with wavelength. The higher value of refractive index in the UV and blue green region is due to band gap and defect state absorption of $\mathrm{ZnO}$ film [10]. The minimum value of extinction coefficient indicates that the $\mathrm{ZnO}$ film has low dielectric loss and this loss will increase with increasing wavelength [10]. The dispersion parameter $A$ and $B$ are obtained using Sellmeier equation as $[10,19]$

$$
\frac{1}{n^{2}-1}=-\frac{A}{\lambda^{2}}+B
$$

Where $A$ and $B$ are slope and intercept of the plot of $\left(n^{2}-1\right)^{-1}$ vs. $\left(\lambda^{2}\right)^{-1}$. The plot is shown in Fig. 5 and least square fit of $\mathrm{A}$ and $\mathrm{B}$ are listed in table 1.

Table 1: Values of ' $n$ ' Extinction coefficient, and least square fit of $A$ and $B$

\begin{tabular}{lllllc}
\hline Thickness of sample $(\mathrm{nm})$ & $\begin{array}{l}\text { Refractive index } \\
(\mathrm{n})\end{array}$ & $\begin{array}{l}\text { Extinction } \\
\text { coefficient }\end{array}$ & $\mathrm{A}\left(\mu \mathrm{m}^{-2}\right)$ & $\mathrm{B}$ & Band gap (eV) \\
\hline $\begin{array}{l}\text { 290nm (SEM Micrograph) } \\
280 \mathrm{~nm} \text { (Envelope method) }\end{array}$ & & $0.28-0.64$ & 0.036 & 0.44 & 3.02 \\
\hline
\end{tabular}

For $\lambda=\infty, n_{\infty}=\left(1+\frac{1}{B}\right)^{1 / 2}$

For longer wavelength, $n_{\infty} \sim 1.86$ which gives $B=$ 0.4 . This value of $B$ for the long wavelength region is well matched with a fitted value as listed in the table.

The value of $\mathrm{n}$ decreases slowly with increasing the wavelength beyond $600 \mathrm{~nm}$. This phenomenon is attributed due to the scattering of the light within surface level defect states. The value of extinction coefficient is considerably low in comparison to the value of the refractive index $n$, which confirms the $\mathrm{ZnO}$ film has a low dielectric loss.

\section{Determination of frequency dependence complex dielectric functions of $\mathrm{ZnO}$ film}

The dielectric function of bulk $\mathrm{ZnO}$ has been intensively studied by various group using Spectroscopic Ellipsometer and impedance analyzer $[20,21,22]$. It is known that the exciton created near band edge absorption significantly influences the dielectric function of nanostructured $\mathrm{ZnO}$. The dielectric properties of this material in visible region offer much information related to impurities, defects and space charge carrier transport. Grain size and surface roughness have the critic role to tune the dielectric function of $\mathrm{ZnO}$ 
films in visible regime. SEM micrograph shows the polycrystalline large number of nano grains with sufficient voids and porosity. The dielectric function of $\mathrm{ZnO}$ primarily depends on the dielectric behavior of grain and grain boundary defect states. The optical constants or dielectric function of nanostructured $\mathrm{ZnO}$ is essentially depends on the band gap of this material. The electronic excitation spectrum of a thin film is generally described in terms of complex electronic dielectric function given below $[10,18]$.

$$
\epsilon(w)=\epsilon_{1}(w)+i \epsilon_{2}(w)
$$

The real part and imaginary part of the complex dielectric function contains the desired information of nanostructured films. The real part and imaginary part of dielectric functions are associated with refractive index $\mathrm{n}$ and extinction coefficient $\mathrm{k}$ by the following formula.

$$
\begin{gathered}
\epsilon_{1}=n^{2}-\mathrm{k}^{2} \ldots(10) \\
\epsilon_{2}=2 \mathrm{nk} \quad \ldots(11)
\end{gathered}
$$

Fig.6 depicts the real and imaginary part of the complex dielectric function of nanostructured $\mathrm{ZnO}$ film. The real part of dielectric function is higher than the imaginary part which confirms the film has reasonably good transparency. $\mathrm{ZnO}$ consists with singly and doubly charged oxygen vacancies $\left(\mathrm{Vo}^{+}\right.$, $\mathrm{Vo}^{++}$) in mid gap states. The optical excitation of the carriers in the valence band discharged the defect levels to increase the conductivity of the film $[22,23]$ by visible illumination and reduced the carrier scattering center [11]. The carrier generation and passivation of defect states under high frequency illumination is increased the real part of the dielectric function, whereas the imaginary part of dielectric function is substantially reduced which indicates the low dielectric loss of this material.

\section{CONCLUSION}

To summarize, a moderately high transmittance undoped $\mathrm{ZnO}$ film was prepared on glass substrate using aged precursor solution of acetate by spin coating technique. Optical band gap and film thickness were measured envelope method applied on interference fringes of the transmittance spectrum invisible region. The film showed the band gap value lies in the UV region $\left(\mathrm{E}_{\mathrm{g}}=3.02 \mathrm{eV}\right)$ and the film thickness was measured $375 \mathrm{~nm}$ by envelop method. Further the calculated thickness was validated by a cross-section view of SEM image of nanostructured $\mathrm{ZnO}$ film. The measured thickness using nondestructive envelope method is nearly consistent with thickness measured from SEM micrograph image. The optical constant such as complex refractive index and dielectric functions were measured in the UV-visible region. The real part of the refractive index decreases with increasing wavelength whereas the imaginary part is increasing. The high value of refractive index at lower wavelength and wavelength dependency primarily depends on the absorption and scattering due to deep levels and sallow level defect states. The visible illumination controls the midgap states by discharging the oxygen vacancies which reduced the carrier scattering center. The complex dielectric function of this material is substantially dependent on the frequency of the light used. The exitonic emission or carrier generation greatly enhanced the real part of dielectric and low dielectric loss.

\section{ACKNOWLEDGEMENTS}

Technology Innovation Center, Sungkyunkwan University, Suwon 440-746, Korea is acknowledged for SEM micrograph. Prof. Shankar Prasad Shrestha is acknowledged for his valuable suggestion and UV visible spectroscopy.

\section{REFERENCES}

[1] Mridha, S. and Basak, D. Aluminium doped $\mathrm{ZnO}$ films: electrical, optical and photoresponse studies. Journal of Physics D: Applied Physics, 40: 6902 (2007).

[2] Mridha, S. and Basak, D. Effect of thickness on the structural, electrical and optical properties of $\mathrm{ZnO}$ films. Materials research bulletin, 42: 875882 (2007).

[3] Li, H.; Wang, J.; Liu, H.; Yang, C.; Xu, H.; Li, X. and Cui, H. Sol-gel preparation of transparent zinc oxide films with highly preferential crystal orientation. Vacuum, 77: 57-62 (2004).

[4] Shrestha, S. P.; Ghimire, R.; Nakarmi, J. J.; Kim, Y. S.; Shrestha, S.; Park, C. Y. and Boo, J. H. Properties of $\mathrm{ZnO}$ : Al films prepared by spin coating of aged precursor solution. Bulletin of the Korean Chemical Society, 31: 112-115 (2010).

[5] Li, H.; Wang, J.; Liu, H.; Zhang, H. and Li, X. Zinc oxide films prepared by sol-gel method. Journal of Crystal Growth, 275: e943e946 (2005).

[6] Yacine, A.; Boubaker, B.; Brahim, G. and Said, B. Structural, optical and electrical properties of 
zinc oxide thin film deposited by a spray pyrolysis technique. Journal of Semiconductors, 36: 013002 (2015).

[7] Wang, J.; Qi, Y.; Zhi, Z.; Guo, J.; Li, M. and Zhang, Y. A self-assembly mechanism for sol-gel derived $\mathrm{ZnO}$ thin films. Smart Materials and Structures, 16: 2673 (2007).

[8] Kayani Z. N.; Siddiq M.; Riaz S. and Nasim S. Optical, magnetic, and structural properties of $\mathrm{Cr}$ Doped $\mathrm{ZnO}$ thin films by sol-gel dip coating technique. Material research express 4: 096403 (2017).

[9] Paul, G. K. and Sen, S. K. Optical properties of some sol-gel-derived gallium-doped $\mathrm{ZnO}$ films. Materials Letters, 57: 959-963 (2002).

[10] Bedia, A.; Bedia, F. Z.; Aillerie, M.; Maloufi, N.; and Benyoucef, B. Influence of the thickness on optical properties of sprayed $\mathrm{ZnO}$ hole-blocking layers dedicated to inverted organic solar cells. Energy Procedia, 50: 603-609 (2014).

[11] Ghimire, R. R. and. Raychaudhuri, A. K. High performance thin film transistor (flex-TFT) with textured nanostructure $\mathrm{ZnO}$ film channel fabricated by exploiting electric double layer gate insulator. Applied Physics Letters, 110: 052105 (2017).

[12] Jin, Y.; Song, B.; Jia, Z.; Zhang, Y.; Lin, C.; Wang, $X$. and Dai, S. Improvement of Swanepole method for deriving the thickness and the optical properties of chalcogenide thin films. Optic express, 25: 440-451 (2017).

[13] Xie, G. C.; Fang, L.; Peng, L. P.; Liu, G. B.; Ruan, H. B.; Wu, F. and Kong, C. Y. Effect of Indoping on the optical constants of $\mathrm{ZnO}$ thin films. Physics Procedi, 32: 651-657 (2012).

[14] Yu, X.; Ma, J.; Ji, F.; Wang, Y.; Cheng, C. and $\mathrm{Ma}, \mathrm{H}$. Thickness dependence of properties of $\mathrm{ZnO}: \mathrm{Ga}$ films deposited by $\mathrm{rf}$ magnetron sputtering. Applied Surface Science, 245: 310-315 (2005).

[15] Chang, J. F. and Hon, M. H. The effect of deposition temperature on the properties of Aldoped Zinc oxide thin films. Thin Solid Films, 386: 79-86 (2001).

[16] Smith, A. Pyrosol deposition of $\mathrm{ZnO}$ and $\mathrm{SnO}_{2}$ based thin films: the interplay between solution chemistry, growth rate and film morphology. Thin Solid Films, 376: 47-55(2000).

[17] Swanepoel, R. Determination of the thickness and optical constants of amorphous silicon. Journal of Physics E: Scientific Instruments, 16: 1214 (1983).

[18] Yan, X.; Zhu, L.; Zhou, Y.; Yiwen, E.; Wang, L.; and $\mathrm{Xu}, \mathrm{X}$. Dielectric property of $\mathrm{MoS}_{2}$ crystal in terahertz and visible regions. Applied optics, 54: 6732-6736 (2015).
[19] Wemple, S. H. and DiDomenico, Jr. M., Behavior of the Electronic Dielectric Constant in Covalent and Ionic Materials. Physical Review B, 3: 13381351 (1971).

[20] Li, X. D.; Chen, T. P.; Liu, P.; Liu, Y.; Liu, Z.; and Leong, K. C. A study on the evolution of dielectric function of $\mathrm{ZnO}$ thin films with decreasing film thickness. Journal of Applied Physics, 115: 103512 (2014).

[21] Miao, L.; Tanemura, S.; Tanemura, M.; Lau, S. P. and Tay, B. K. Thickness-dependent optical properties of $\mathrm{ZnO}$ thin films. Journal of Materials Science: Materials in Electronics, 18: $343-346$ (2007).

[22] Fujiwara, H. and Kondo, M. Effects of carrier concentration on the dielectric function of $\mathrm{ZnO}$ : $\mathrm{Ga}$ and $\mathrm{In}_{2} \mathrm{O}_{3}$ : $\mathrm{Sn}$ studied by spectroscopic ellipsometry: analysis of free-carrier and bandedge absorption. Physical Review B, 71: 075109 (2005).

[23] Xiong, K.; Du, Y.; Tse, K. and Robertson, J. Defect states in the high-dielectric-constant gate oxide $\mathrm{HfSiO}_{4}$. Journal of Applied Physics, 101: 024101 (2007).

[24] Oing, D.; Geller, M.; Stahl, L.; Kerski, J.; Lorke, A. and Wöhrl, N. Defect spectroscopy on the dielectric material aluminium oxide. Scientific report, 10: 12533 (2020). 